\title{
Cephalopod paralarvae assemblages in Hawaiian Islands waters
}

\author{
John R. Bower ${ }^{1 *}$, Michael P. Seki ${ }^{2}$, Richard E. Young ${ }^{3}$, Keith A. Bigelow ${ }^{4}$, \\ Jed Hirota ${ }^{3}$, Pierre Flament ${ }^{3}$
}

\author{
${ }^{1}$ Faculty of Fisheries, Hokkaido University, 3-1-1 Minato-cho, Hakodate, Hokkaido, 041-8611, Japan \\ ${ }^{2}$ Honolulu Laboratory, Southwest Fisheries Science Center, National Marine Fisheries Service, NOAA, 2570 Dole Street, \\ Honolulu, Hawaii 96822-2396, USA \\ ${ }^{3}$ Department of Oceanography, University of Hawaii at Manoa, 1000 Pope Road, Honolulu, Hawaii 96822, USA \\ ${ }^{4}$ Oceanic Fisheries Programme, Secretariat of the Pacific Community, BP D5, 98848 Noumea Cedex, New Caledonia
}

\begin{abstract}
The distribution and abundance of cephalopod paralarvae near the Hawailan Islands are described. Paralarvae were collected during 5 plankton surveys in 1991 to 1993 . The 404 tows at 59 stations collected 10375 paralarvae from 21 families and 57 species. The most numerous families were the Ommastrephidae $(23 \%$ of total catch), Pyroteuthididae $(17 \%)$, Enoploteuthididae $(16 \%)$, Onychoteuthididae $(14 \%)$, and Chtenopterygidae $(8 \%)$. The most numerous species were Ommastrephes bartramii $(18 \%)$, Pterygioteuthis microlampas $(15 \%)$, Chtenopteryx sicula $(8 \%)$, and Onychoteuthis compacta $(6 \%)$. Analysis of paralarval distribution patterns identified 2 paralarval assemblages: 'island associated' and 'oceanic' The 15 'island-associated' species showed increased paralarval abundance near the islands, suggesting preferential spawning in this area. Epipelagic nearshore spawners included Onychoteuthis sp. C, Sthenoteuthis oualaniensis, and Nototodarus hawailensis. Mesopelagic nearshore spawners included 3 reported members of the Hawailan Mesopelagic Boundary Community (Abralia trigonura, Liocranchia reinhardti, and Chiroteuthis picteti) and 3 probable new members (Liocranchia valdiviae, Histioteuthis hoylei and Enoploteuthis jonesi).
\end{abstract}

KEY WORDS: Paralarvae Cephalopod Hawaijan Islands - Distribution · Assemblage

\section{INTRODUCTION}

Cephalopod paralarvae are more numerous and more easily sampled than adults. Knowledge of paralarval distribution and abundance patterns is useful for determining when and where adults spawn, particularly for species whose adults are difficult to catch. Knowledge of the early life stages can also help in understanding cephalopod population dynamics and in developing stock-recruitment models for commercially important species (Vecchione 1987). Yet, despite their importance, the early life stages of most species are seldom studied and poorly understood (Boyle 1990).

A diverse community of cephalopods occurs near the Hawaiian Islands (Berry 1914, Young 1978). It includes

\footnotetext{
•E-mail: akaika@pop.fish.hokudai.ac.jp
}

inhabitants of the mesopelagic-boundary region (Reid et al. 1991, Young 1995), one of the most poorly investigated habitats in the ocean. Benthic adults are particularly difficult to sample because the steep and jagged seafloor near the islands restricts benthic trawling. As a result, the species composition and distribution of cephalopods around the islands remain uncertain.

To date, the paralarval distribution patterns of only 1 cephalopod species (Ommastrephes bartramii) near the Hawaiian Islands have been described (Young \& Hirota 1990, Bower 1994, 1996). In this paper, we present the paralarval distribution patterns of 58 cephalopod species collected near the islands during 5 surveys in 1991 to 1993 . We then identify species-assemblage groups based on capture distance from the islands and compare onshore-offshore abundance patterns to infer which species may spawn preferentially near the islands. 


\section{MATERIALS AND METHODS}

Sampling. Five plankton surveys were conducted near the Hawaiian Islands to collect cephalopod paralarvae. Stations were located 9 to $1169 \mathrm{~km}$ windward (northeast) and 11 to $256 \mathrm{~km}$ leeward (southwest) of the islands. Samples were collected aboard the Hokkaido University ship 'Hokusei Maru' (HM) during 6 to 15 February 1991 (HM-91 survey; 7 stations, 3 to 5 tows station ${ }^{-1}$ ), 5 to 26 February 1992 (HM-92 survey; 16 stations, 7 to 10 tows station ${ }^{-1}$ ), and 4 to 19 February 1993 (HM-93 survey; 12 stations, 7 to 10 tows station ${ }^{-1}$ ), and aboard the NOAA ship 'Townsend Cromwell' (TC) during 5 to 20 February 1991 (TC-91 survey; 10 stations, 1. to 7 tows station ${ }^{-1}$ ) and 22 March to 7 April 1992 (TC92 survey; 14 stations, 4 to 8 tows station ${ }^{-1}$ ). The standard sampling procedure in all surveys was to conduct 30 min open oblique tows from $100 \mathrm{~m}$ depth to the surface during the day. This depth range was chosen to sample through the depths known for paralarval Ommastrephes bartramii. Species with paralarval distributions deeper than this depth range will thus be underrepresented. In total, 404 tows were conducted at 59 stations. All $\mathrm{HM}$ surveys used a $4 \mathrm{~m}^{2}$ ring net equipped with $0.505 \mathrm{~mm}$ mesh, a General Oceanics flowmeter, and a Benthos time-depth recorder (TDR). The TC-91 survey also used a $4 \mathrm{~m}^{2}$ ring net. The TC-92 survey used a $2 \mathrm{~m}^{2}$ ring net for 94 tows and a $4 \mathrm{~m}^{2}$ ring net for 4 tows. TC nets carried a Wildlife Computers TDR. Bower (1996) described the sampling procedure in detail.

Net collections were fixed at sea in 4 to $6 \%$ buffered formalin for 3 to $6 \mathrm{~h}$, then preserved in 50\% isopropyl alcohol. During the HM-91 survey, paralarvae were sorted from the plankton at sea with dissecting microscopes. Samples from subsequent TC and HM surveys were sorted on shore. Paralarvae were identified to genus and species level with the aid of published figures and descriptions (Harman \& Young 1985, Young \& Harman 1985, 1987, 1989 , Young et al. 1985, Young \& Hirota 1990, Young 1991). In some genera, more than 1 species was present, but identification was not possible either because a growth series connecting paralarvae to known adults was not available or the adults were known but undescribed. In such cases, species were given letter designations. Abundances of each species were standardized (individuals per $50 \mathrm{~m}^{2}$ of sea surface sampled) for each tow (Smith \& Richardson 1977). Station abundances were calculated as the average of all station tows.

Assemblage groups. Assemblage groups were determined with the 2-way indicator species analysis program TWINSPAN (Hill 1979, Gauch \& Whittaker 1981) using PC-ORD software (McCune \& Mefford 1997). Analyses are based on abundance data from all surveys for species with total catches greater than 15. Abundances at each station were used for comparisons of onshore-offshore distribution to determine which species preferentially spawn near the islands (Spearman rank test, $p<0.05$ ).

\section{RESULTS}

\section{Family and species composition}

The 404 tows at 59 stations collected 10375 paralarvae from 21 families and 58 species (Table 1). The most numerous families were the Ommastrephidae $(23 \%$ of total catch), Pyroteuthididae (17\%), Enoploteuthididae (16\%), Onychoteuthididae (14\%), and Chtenopterygidae $(8 \%)$. The most numerous species were Ommastrephes bartramii $(18 \%)$, Pterygioteuthis microlampas $(15 \%)$, Chtenopteryx sicula $(8 \%)$, and Onychoteuthis compacta $(6 \%)$. Interannual catch rates for most species varied widely. Catches of the most abundant species showed a general decline over the 3 survey years. Results for the more frequently taken taxa are presented below.

\section{Species distribution: squids}

A total of 8848 squids from 16 families and 44 species were collected.

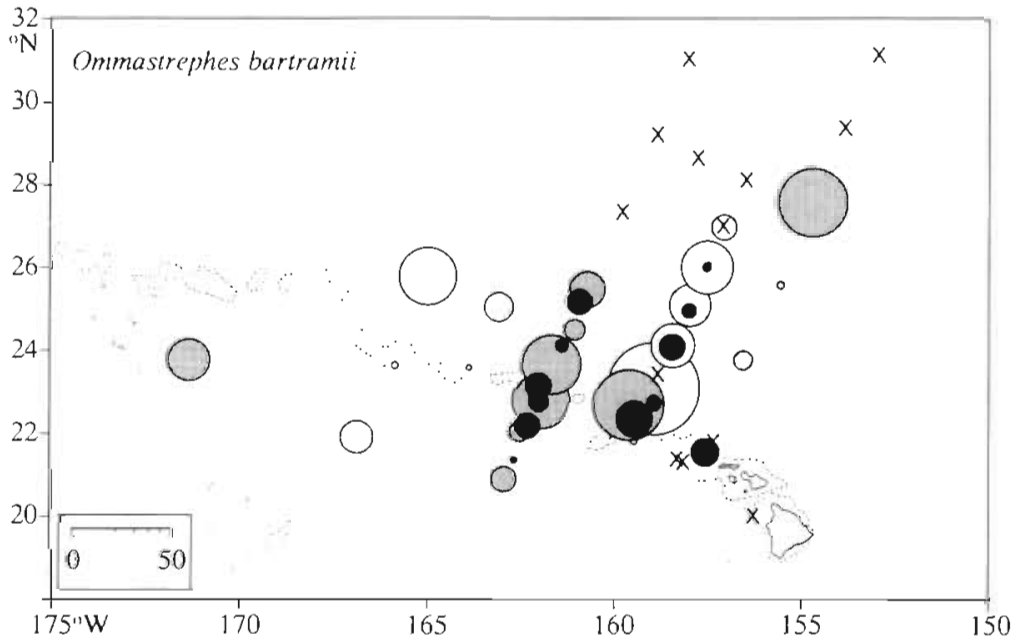

Fig. 1. Distribution and abundance of Ommastrephes bartramii paralarvae collected near the Hawaiian Islands during 5 cruises in 1991 to 1993. Circles represent mean station abundance (ind./50 $\mathrm{m}^{2}$ ). White circles: 1991 catches; gray circles: 1992 catches; black circles: 1993 catches. Circle area represents catch abundance, $X$ : no catch 
Family Ommastrephidae. Ommastrephes bartramii was the most numerous cephalopod species collected, composing $18 \%$ of the cephalopod catch and $80 \%$ of the ommastrephid catch; catches occurred at 41 stations (Fig. 1), and $40 \%$ were collected more than $200 \mathrm{~km}$ offshore. Hyaloteuthis pelagica was also widely distributed, occurring at 37 stations; $35 \%$ were collected more than $200 \mathrm{~km}$ offshore. Catches of the other 2 ommastrephids represented in the collections occurred farther inshore. Sthenoteuthis oualaniensis was collected at 26 stations (Fig. 2); $40 \%$ occurred less than $25 \mathrm{~km}$ offshore. Nototodarus hawaiiensis was collected at 15 stations; $54 \%$ occurred less than $25 \mathrm{~km}$ offshore.
Family Pyroteuthididae. All 3 pyroteuthid species were widely distributed. Pterygioteuthis microlampas composed $89 \%$ of the pyroteuthid catch and was the most commonly collected cephalopod species, occurring in $68 \%$ of the tows and at 56 stations (Fig. 3); $41 \%$ occurred more than $200 \mathrm{~km}$ offshore. Pterygioteuthis giardi was collected at 26 stations; $54 \%$ occurred more than $200 \mathrm{~km}$ offshore. Pyroteuthis addolux was collected at 17 stations; $81 \%$ occurred more than $200 \mathrm{~km}$ offshore.

Family Enoploteuthididae. Abraliopsis sp. A (Young \& Harman 1985) was collected at 46 stations (Fig. 4) and composed $30 \%$ of the enoploteuthid catch; $66 \%$ occurred more than $200 \mathrm{~km}$ offshore. Enoploteuthis

Table 1. Cephalopod paralarvae collected near the Hawaiian Islands during 5 surveys in 1991 to 1993 . Numbers for higher taxa include specimens that could not be further identified. HM: 'Hokusei Maru'; TC: 'Townsend Cromwell'. 91: 1991; 92: 1992; 93: 1993. Mean abundance: ind. $/ 50 \mathrm{~m}^{2}$ NE: distance $(\mathrm{km})$ from archipelago to northeasternmost point of capture; SW: distance $(\mathrm{km})$ from archipelago to southwesternmost point of capture. This point will lie windward (northeast) of the archipelago if no captures were made south of the archipelago. W: windward of the archipelago; L: leeward of the archipelago. na: not applicable

\begin{tabular}{|c|c|c|c|c|c|c|c|c|c|c|c|}
\hline & \multirow[t]{2}{*}{ HM-91 } & \multirow[t]{2}{*}{ TC-91 } & \multirow[t]{2}{*}{ HM-92 } & \multirow[t]{2}{*}{ TC-92 } & \multirow[t]{2}{*}{ HM-93 } & \multirow[t]{2}{*}{ Total } & \multirow{2}{*}{$\begin{array}{c}\text { Positive } \\
\text { tows } \\
(\%)\end{array}$} & \multirow{2}{*}{$\begin{array}{l}\text { Positive-tow } \\
\text { mean abund- } \\
\text { ance }(\mathrm{SD})\end{array}$} & \multicolumn{2}{|c|}{$\begin{array}{l}\text { Offshore } \\
\text { range }\end{array}$} & \multirow{2}{*}{$\begin{array}{l}\% \text { collected } \\
\quad<50 \mathrm{~km} \\
\text { offshore }\end{array}$} \\
\hline & & & & & & & & & & SW & \\
\hline Order Octopoda & 29 & 333 & 82 & 17 & 128 & 589 & & & & & \\
\hline Family Argonautidae & 2 & 3 & 3 & 2 & 4 & 14 & & & & & \\
\hline Argonauta argo & 2 & 3 & 3 & 2 & 4 & 14 & 3 & $0.8(0.4)$ & $486-W$ & $14-W$ & 21 \\
\hline Family Bolitaenidae & 1 & 1 & 6 & 0 & 9 & 17 & & & & & \\
\hline Eledonella pygmaea & 1 & 1 & 6 & 0 & 9 & 17 & 3 & $0.9(0.3)$ & $736-\mathrm{W}$ & $204-W$ & 0 \\
\hline Family Octopodidae & 26 & 325 & 70 & 12 & 111 & 544 & & & & & \\
\hline Octopus cyanea & 14 & 250 & 38 & 3 & 83 & 388 & 18 & $4.3(7.9)$ & $264-W$ & $256-\mathrm{L}$ & 82 \\
\hline Octopus ornatus & 0 & 2 & 3 & 0 & 0 & 5 & 1 & $1.0(0.3)$ & $26 \cdot W$ & $9-W$ & 100 \\
\hline Octopus Type $A^{d}$ & 1 & 3 & 0 & 0 & 2 & 6 & 1 & $1.0(0.6)$ & $26-W$ & $113-\mathrm{L}$ & 83 \\
\hline Octopus Type $\mathrm{B}^{\mathrm{d}}$ & 0 & 13 & 2 & 4 & 7 & 26 & 3 & $2.0(1.1)$ & $143-W$ & $113-\mathrm{L}$ & 73 \\
\hline Octopus Type $\mathrm{C}^{\mathrm{d}}$ & 1 & 11 & 1 & 1. & 5 & 19 & 4 & $1.0(0.5)$ & $264-W$ & $34-\mathrm{L}$ & 84 \\
\hline Octopus Type $\mathrm{D}^{\circ}$ & 1 & 1 & 1 & 1 & 0 & 4 & 1 & $1.2(0.4)$ & $59-W$ & $126-\mathrm{L}$ & 50 \\
\hline Octopus Type $E^{\alpha}$ & 4 & 18 & 1 & 2 & 0 & 25 & 3 & $2.2(2.4)$ & $59-W$ & $11-\mathrm{L}$ & 92 \\
\hline Octopus Type $\mathrm{H}^{\star}$ & 2 & 14 & 8 & 1 & 13 & 38 & 7 & $1.0(0.6)$ & $351-W$ & $113-\mathrm{L}$ & 66 \\
\hline Octopus Type Id & 3 & 13 & 9 & 0 & 1 & 26 & 5 & $1.1(0.6)$ & $238-W$ & $34-\mathrm{L}$ & 73 \\
\hline Family Tremoctopodıdae & 0 & 4 & 3 & 3 & 4 & 14 & & & & & \\
\hline Tremoctopus violaceus & 0 & 4 & 3 & 3 & 4 & 14 & 3 & $1.1(0.5)$ & $762-W$ & $215-\mathrm{L}$ & 14 \\
\hline Order Sepioidea & 1 & 1 & 0 & 0 & 0 & 2 & & & & & \\
\hline Family Sepiolidae & 1 & 1 & 0 & 0 & 0 & 2 & & & & & \\
\hline Euprymna scolopes & 1 & 1 & 0 & 0 & 0 & 2 & 1 & $1.0(0.1)$ & $26-W$ & $34-\mathrm{L}$ & 100 \\
\hline Order Teuthida & 1790 & 1874 & 2263 & 1293 & 1628 & 8848 & & & & & \\
\hline Family Ancistrocheiridae & 3 & 9 & 4 & 7 & 2 & 25 & & & & & \\
\hline Ancistrocheirus lesueurii & 3 & 9 & 4 & 7 & 2 & 25 & 5 & $1.1(0.8)$ & $721-W$ & $212-\mathrm{L}$ & 36 \\
\hline Family Brachioteuthididae & 101 & 73 & 117 & 45 & 56 & 392 & & & & & \\
\hline Brachioteuthis sp. & 101 & 73 & 117 & 45 & 56 & 392 & 42 & $2.1(2.2)$ & 1169-W & $256-\mathrm{L}$ & 43 \\
\hline Family Chiroteuthididae & 31 & 20 & 48 & 18 & 12 & 129 & & & & & \\
\hline Chiroteuthis picteti & 3 & 10 & 5 & 0 & 3 & 21 & 4 & $1.3(0.6)$ & $183-W$ & $113-\mathrm{L}$ & 57 \\
\hline Chiroteuthis sp. nov. ${ }^{\mathrm{b}}$ & 27 & 7 & 43 & 16 & 8 & 101 & 17 & $1.2(0.9)$ & $762-W$ & $126-\mathrm{L}$ & 28 \\
\hline Grimalditeuthis bonplandi & 0 & 3 & 0 & 2 & 1 & 6 & 1 & $1.4(0.6)$ & $1169-\mathrm{W}$ & $113-\mathrm{L}$ & 50 \\
\hline Planctoteuthis danae & 1 & 0 & 0 & 0 & 0 & 1 & 0.2 & 1.6 (na) & $106-W$ & $106-W$ & 0 \\
\hline Family Chtenopterygidae & 192 & 103 & 272 & 65 & 141 & 773 & & & & & \\
\hline Chtenopteryx sicula & 192 & 103 & 272 & 65 & 141 & 773 & 59 & $2.7(2.9)$ & $1169-W$ & $256-\mathrm{L}$ & 26 \\
\hline
\end{tabular}


Table 1 (continued)

\begin{tabular}{|c|c|c|c|c|c|c|c|c|c|c|c|}
\hline & \multirow[t]{2}{*}{ HM-91 } & \multirow[t]{2}{*}{ TC-91 } & \multirow[t]{2}{*}{$\mathrm{HC}-92$} & \multirow[t]{2}{*}{ TC-92 } & \multirow[t]{2}{*}{ HM-93 } & \multirow[t]{2}{*}{ Total } & \multirow{2}{*}{$\begin{array}{l}\text { Positive } \\
\text { tows } \\
(\%)\end{array}$} & \multirow{2}{*}{$\begin{array}{l}\text { Positive-tow } \\
\text { mean abund- } \\
\text { ance (SD) }\end{array}$} & \multicolumn{2}{|c|}{$\begin{array}{c}\text { Offshore } \\
\text { range }\end{array}$} & \multirow{2}{*}{$\begin{array}{c}\% \text { collected } \\
<50 \mathrm{~km} \\
\text { offshore }\end{array}$} \\
\hline & & & & & & & & & & & \\
\hline Family Cranchiidae & 96 & 214 & 209 & 32 & 188 & 739 & & & & & \\
\hline Cranchia scabra & 29 & 28 & 95 & 7 & 69 & 228 & 34 & $1.2(0.8)$ & $736-W$ & $256-\mathrm{L}$ & 29 \\
\hline Galiteuthis pacifica & 1 & 0 & 5 & 1 & 5 & 12 & 3 & $0.7(0.3)$ & $1169-W$ & 9-W & 25 \\
\hline Helicocranchia sp. $\mathrm{B}^{\mathrm{C}}$ & 40 & 59 & 15 & 4 & 52 & 170 & 14 & $3.0(4.9)$ & $979-W$ & $207-\mathrm{L}$ & 43 \\
\hline Leachia pacifica & 16 & 25 & 25 & 12 & 16 & 94 & 18 & $1.1(0.7)$ & $721-W$ & $256-\mathrm{L}$ & 49 \\
\hline Liocranchia reinhardti & 6 & 67 & 50 & 4 & 25 & 152 & 22 & $1.3(1.3)$ & $471-W$ & $256-\mathrm{L}$ & 70 \\
\hline Liocranchia valdiviae & 4 & 33 & 18 & 4 & 19 & 78 & 14 & $1.2(1.0)$ & $721-W$ & $207-\mathrm{L}$ & 62 \\
\hline Megalocranchia fisheri & 0 & 2 & 1 & 0 & 2 & 5 & 1 & $0.8(0.2)$ & $230-W$ & $30-\mathrm{L}$ & 60 \\
\hline Family Cycloteuthididae & 0 & 1. & 0 & 0 & 0 & 1 & & & & & \\
\hline Cycloteuthis sirventi & 0 & 1 & 0 & 0 & 0 & 1 & 0.2 & 0.8 (na) & $14-W$ & $14-W$ & 100 \\
\hline Family Enoploteuthididae & 357 & 351 & 416 & 121 & 270 & 1515 & & & & & \\
\hline Abralia astrosticta & 0 & 6 & 0 & 0 & 1 & 7 & 1 & $1.7(1.1)$ & $71-W$ & $14-W$ & 86 \\
\hline Abralia trigonura & 108 & 185 & 34 & 17 & 27 & 371. & 20 & $4.4 \quad(5.1)$ & $264-W$ & $113-\mathrm{L}$ & 88 \\
\hline Abraliopsis pacificus & 39 & 39 & 82 & 39 & 80 & 279 & 38 & $1.5(1.2)$ & $1169-W$ & $256-\mathrm{L}$ & 50 \\
\hline Abraliopsis sp. $A^{\downarrow}$ & 101 & 38 & 180 & 48 & 86 & 453 & 45 & $2.2(2.3)$ & $1169-\mathrm{W}$ & $256-\mathrm{L}$ & 24 \\
\hline Enoploteuthis higginsi & 78 & 67 & 78 & 13 & 52 & 288 & 37 & $1.7(1.6)$ & $721-W$ & $256-\mathrm{L}$ & 39 \\
\hline Enoploteuthis jonesi & 1 & 6 & 8 & 0 & 6 & 21 & 4 & $1.0 \quad(0.6\}$ & $264-W$ & $207-\mathrm{L}$ & 81 \\
\hline Enoploteuthis reticulata & 30 & 10 & 34 & 4 & 15 & 93 & 16 & $1.3(1.4)$ & $736-W$ & $256-\mathrm{L}$ & 27 \\
\hline Family Histioteuthididae & 8 & 26 & 7 & 5 & 8 & 54 & & & & & \\
\hline Histioteuthis hoylei & 0 & 14 & 6 & 0 & 4 & 24 & 3 & $1.6(1.2)$ & $143-W$ & $207-\mathrm{L}$ & 79 \\
\hline Histioteuthis $\mathrm{sp} . \mathrm{B}^{\mathrm{c}}$ & 0 & 3 & 0 & 0 & 1 & 4 & 1 & $1.8(1.6)$ & $230-W$ & $204-W$ & 0 \\
\hline Histioteuthis sp. $\mathrm{C}^{\mathrm{c}}$ & 7 & 9 & 1 & 1 & 3 & 21 & 4 & $1.2(1.0)$ & $581-W$ & $113-\mathrm{L}$ & 33 \\
\hline Histioteuthis sp. $\mathrm{D}^{\mathrm{r}}$ & 1 & 0 & 0 & 4 & 0 & 5 & 1 & $1.3(0.3)$ & $979-W$ & $351-W$ & 0 \\
\hline Family Lepidoteuthididae & 2 & 0 & 0 & 1 & 0 & 3 & & & & & \\
\hline Lepidoteuthis grimaldii & 2 & 0 & 0 & 1 & 0 & 3 & 1 & $1.2(0.3)$ & $955-W$ & $16-W$ & 33 \\
\hline Family Mastigoteuthididae & 0 & 5 & 1 & 0 & 0 & 6 & & & & & \\
\hline Mastigoteuthis famelica & 0 & 5 & 1 & 0 & 0 & 6 & 1 & $0.9(0.2)$ & $183-W$ & $14-W$ & 83 \\
\hline Family Octopoteuthididae & 10 & 9 & 10 & 9 & 12 & 50 & & & & & \\
\hline Octopoteuthis nielseni & 10 & 9 & 10 & 9 & 8 & 46 & 9 & $1.2(0.7)$ & $1169-W$ & $207-\mathrm{L}$ & 30 \\
\hline Taningia danae & 0 & 0 & 0 & 0 & 4 & 4 & 1 & $1.0(0.2)$ & $30-\mathrm{L}$ & $207-\mathrm{L}$ & 50 \\
\hline Family Ommastrephidae & 403 & 210 & 378 & 634 & 519 & 2144 & & & & & \\
\hline Hyaloteuthis pelagica & 22 & 65 & 90 & 34 & 40 & 251 & 27 & $2.1(4.0)$ & $736-\mathrm{W}$ & $256-\mathrm{L}$ & 49 \\
\hline Nototodarus hawaiiensis & 6 & 11 & 11 & 1 & 6 & 35 & 6 & $1.0(0.4)$ & $264-W$ & $113-L$ & 80 \\
\hline Ommastrephes bartramii & 368 & 100 & 242 & 556 & 453 & 1719 & 47 & $8.1(11.9)$ & $721-W$ & $256-\mathrm{L}$ & 34 \\
\hline Sthenoteuthis oualaniensis & 7 & 34 & 35 & 43 & 20 & 139 & 19 & $1.9(2.2)$ & $581-W$ & $256-\mathrm{L}$ & 63 \\
\hline Family Onychoteuthididae & 156 & 444 & 343 & 103 & 251 & 1297 & & & & & \\
\hline Onychoteuthis compacta & 79 & 226 & 114 & 26 & 93 & 538 & 46 & $2.4(3.3)$ & $11.69-W$ & $256-\mathrm{L}$ & 33 \\
\hline Onychoteuthis sp. $\mathrm{B}^{\mathrm{e}}$ & 0 & 3 & 4 & 0 & 7 & 14 & 3 & $0.8(0.3)$ & $264-W$ & $256-\mathrm{L}$ & 64 \\
\hline Onychoteuthis sp. $\mathrm{C}^{\mathrm{e}}$ & 34 & 166 & 117 & 13 & 87 & 417 & 36 & $2.3(2.3)$ & $721-W$ & $256-\mathrm{L}$ & 73 \\
\hline Onykia sp. $\mathrm{A}^{\mathrm{c}}(\mathrm{O}$. carriboea?) & 38 & 47 & 103 & 61 & 58 & 307 & 43 & $1.5(1.1)$ & $721-W$ & $256-\mathrm{L}$ & 36 \\
\hline Onykia sp. $\mathrm{B}^{\mathrm{C}}$ (O. rancureli?) & 5 & 1 & 5 & 3 & 0 & 14 & 3 & $1.5(0.7)$ & 979-W & $16-W$ & 29 \\
\hline Family Pholidoteuthididae & 10 & 11. & 0 & 1 & 2 & 24 & & & & & \\
\hline Pholidoteuthis sp. & 10 & 11 & 0 & 1 & 2 & 24 & 3 & $1.7(1.0)$ & $721-W$ & $22-W$ & 8 \\
\hline Family Pyroteuthididae & 411 & 377 & 419 & 234 & 147 & 1588 & & & & & \\
\hline Pterygioteuthis giardi & 16 & 40 & 35 & 1 & 15 & 107 & 13 & $1.8(1.2)$ & $955-W$ & $215-\mathrm{L}$ & 45 \\
\hline Pterygioteuthis microlampas & 373 & 331 & 360 & 227 & 125 & 1416 & 68 & $5.0 \quad[6.2]$ & $955-W$ & $256-\mathrm{L}$ & 40 \\
\hline Pyroteuthis addolux & 22 & 6 & 21 & 6 & 7 & 62 & 8 & $2.2(3.0)$ & $979-W$ & $215-\mathrm{L}$ & 8 \\
\hline Family Thysanoteuthididae & 10 & 21 & 39 & 18 & 20 & 108 & & & & & \\
\hline Thysanoteuthis rhombus & 10 & 21 & 39 & 18 & 20 & 108 & 18 & $1.2(0.7)$ & $721-W$ & $256-\mathrm{L}$ & 42 \\
\hline Unidentified & 185 & 180 & 371 & 133 & 67 & 936 & & & & & \\
\hline Total cepalopods & 2005 & 2388 & 2716 & 1443 & 1823 & 10375 & & & & & \\
\hline Total species & 45 & 52 & 46 & 41 & 47 & 57 & & & & & \\
\hline
\end{tabular}


higginsi, Abraliopsis pacificus and E. reticulata also had wide latitudinal distributions. Abralia trigonura (Fig. 5) and E. jonesi were collected much closer to shore; $40 \%$ and $67 \%$ of their respective catches occurred less than $25 \mathrm{~km}$ offshore.

Family Onychoteuthididae. Onychoteuthis compacta was collected at 50 stations (Fig. 6) and composed $43 \%$ of the onychoteuthid catch; $59 \%$ occurred more than $200 \mathrm{~km}$ offshore. Onykia sp. A was also widely distributed at 50 stations, but less abundant than O. compacta; 40\% occurred more than $200 \mathrm{~km}$ offshore. Onychoteuthis sp. C (Young \& Harman 1987) was widely distributed at 41 stations (Fig. 7), yet $53 \%$ occurred less than $25 \mathrm{~km}$ offshore. Onychoteuthis sp. B (Young \& Harman 1987) was collected at 9 stations; $50 \%$ occurred less than $25 \mathrm{~km}$ offshore.

Family Chtenopterygidae. Chtenopteryx sicula was the most widely distributed cephalopod collected, occurring at $97 \%$ of the stations (Fig. 8); 57\% were collected more than $200 \mathrm{~km}$ offshore.

Family Cranchiidae. Cranchia scabra was collected at 42 stations (Fig. 9) and composed $31 \%$ of the cranchiid catch; $58 \%$ occurred more than $200 \mathrm{~km}$ offshore. Helicocranchia sp. B was collected at 22 stations and composed $23 \%$ of the cranchiid catch; $50 \%$ occurred more than $200 \mathrm{~km}$ offshore. Leachia pacifica was also widely distributed; catches occurred at 38 stations, and $37 \%$ were collected more than $200 \mathrm{~km}$ offshore. Liocranchia reinhardti and Liocranchia valdiviae occurred closer to shore; 46 and $41 \%$ of their respective catches occurred less than $25 \mathrm{~km}$ offshore.

\section{Species distribution: octopods}

A total of 589 octopods from 4 families and 12 species were collected. The 9 octopodid species composed $92 \%$ of the octopod numeric catch. All octopodids were collected less than $352 \mathrm{~km}$ from the islands; $59 \%$ occurred less than $25 \mathrm{~km}$ offshore. Octopus cyanea was collected at 23 stations (Fig. 10) and composed $72 \%$ of the octopodid numeric catch. Octopus Type H (Young \& Harman 1989) had the furthest offshore distribution of the octopodids; $29 \%$ were collected more than $200 \mathrm{~km}$ off-

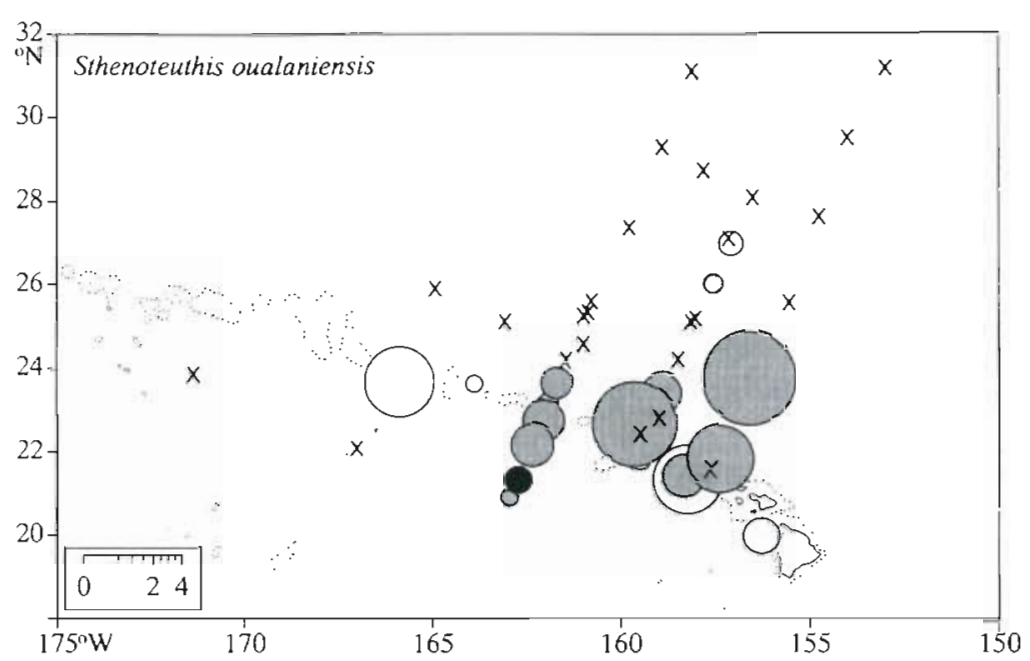

Fig. 2. Distribution and abundance of Sthenoteuthis oualaniensis. Symbols described in Fig. 1

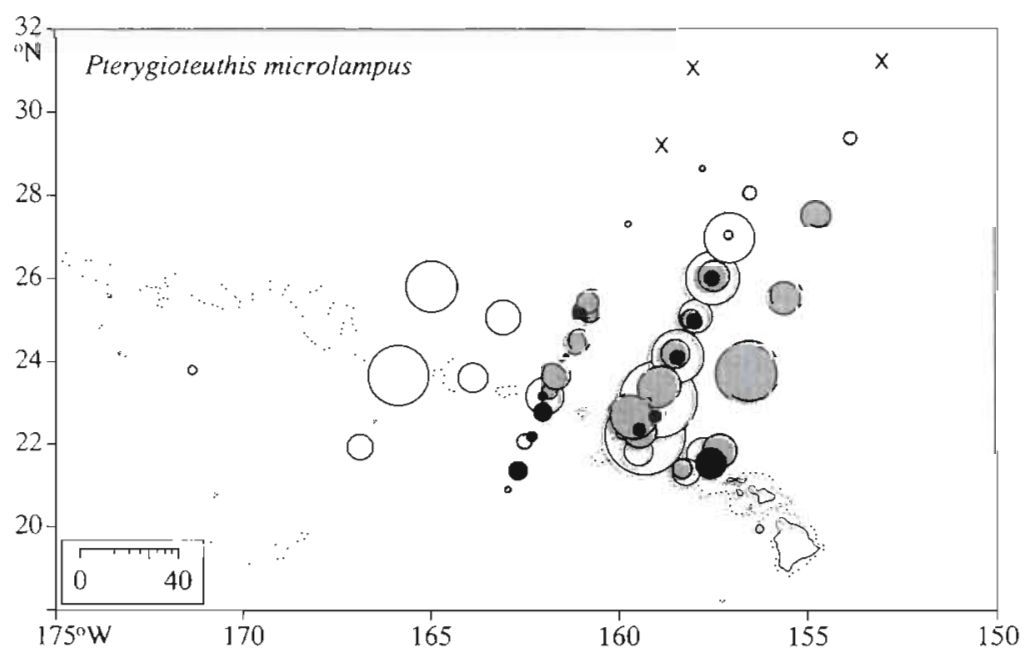

Fig. 3. Distribution and abundance of Pterygioteuthis microlampus. Symbols described in Fig. 1

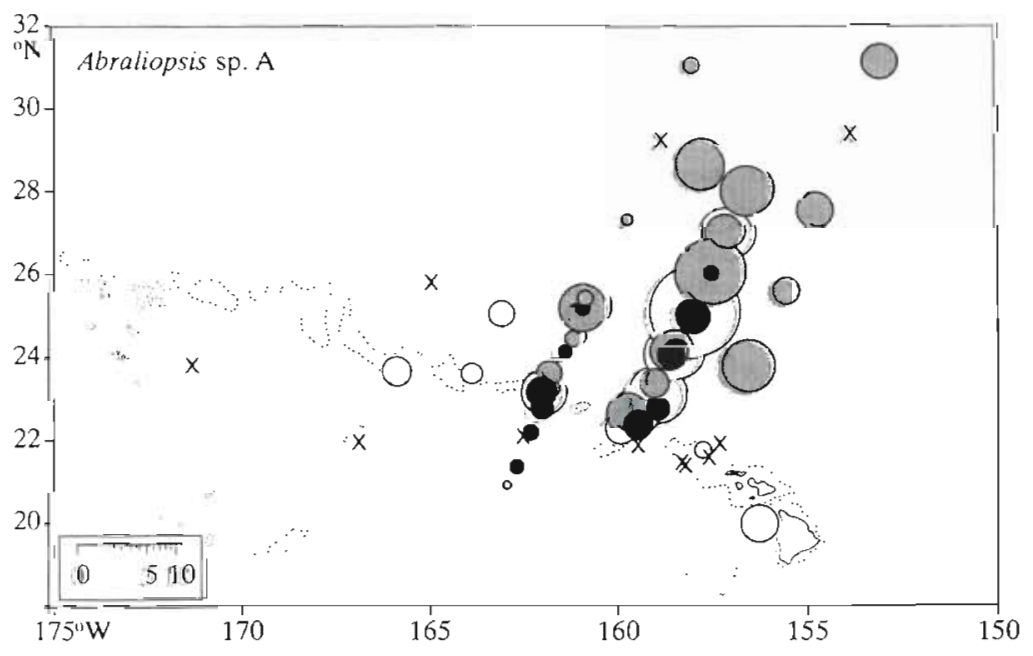

Fig. 4. Distribution and abundance of Abraliopsis sp. A. Symbols described in Fig. 1 


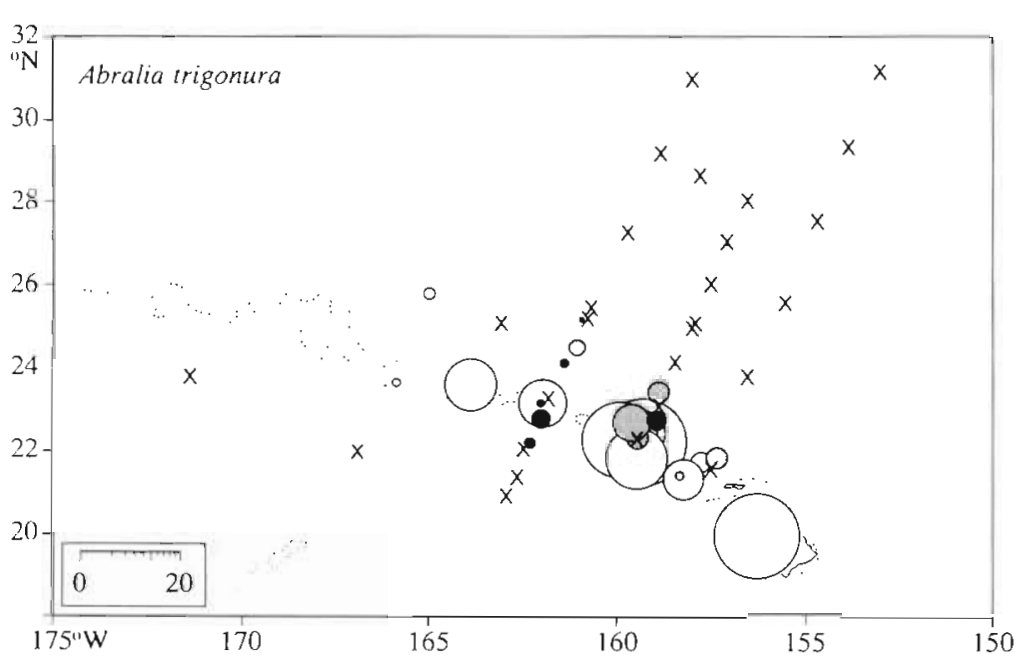

Fig. 5. Distribution and abundance of Abralia trigonura. Symbols described in Fig. 1

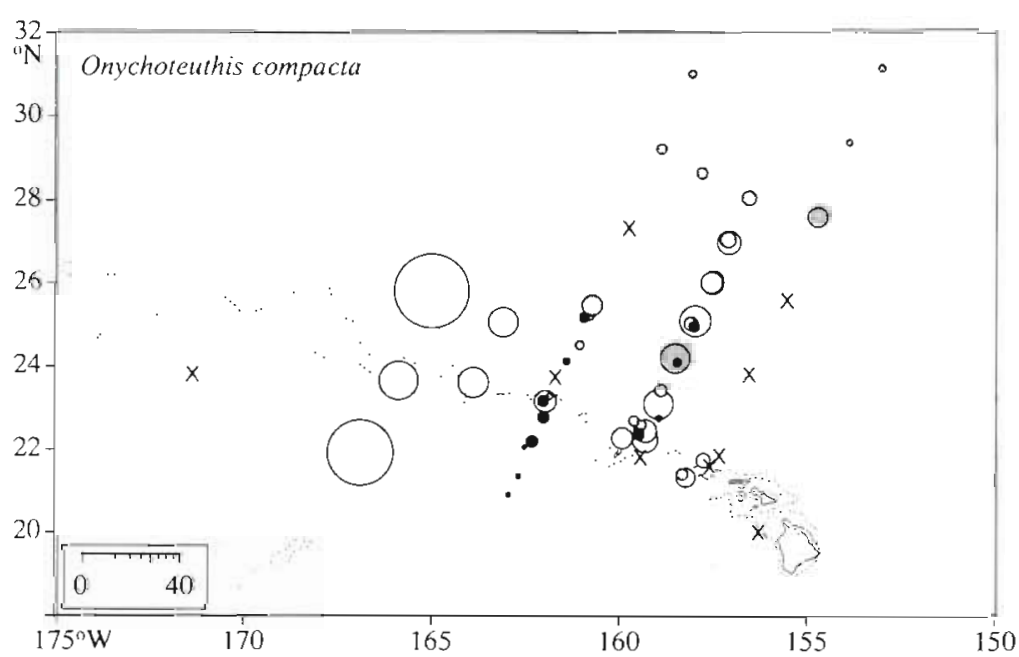

Fig. 6. Distribution and abundance of Onychoteuthis compacta. Symbols described in Fig. 1

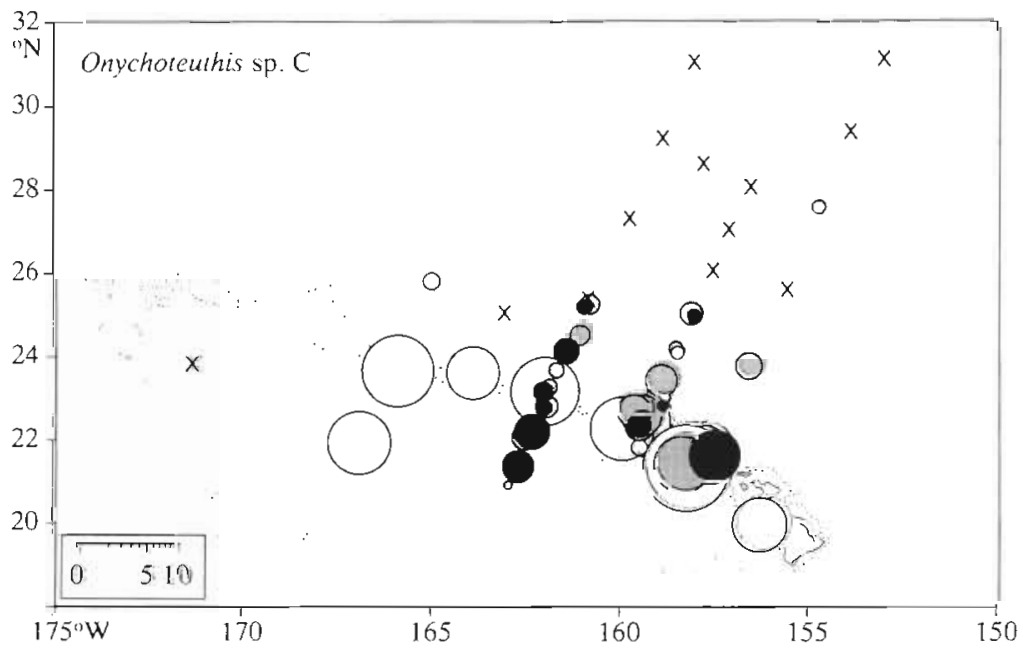

Fig. 7. Distribution and abundance of Onychoteuthis sp. C. Symbols described in Fig. 1 shore. Eledonella pygmaea was the only cephalopod collected exclusively offshore; catches occurred at 8 windward stations north of $25^{\circ} 02^{\prime} \mathrm{N}$ greater than $200 \mathrm{~km}$ offshore. Tremoctopus violaceus was collected at 11 stations and had the widest latitudinal range of all octopods $\left(21^{\circ} 20^{\prime}\right.$ to $29^{\circ} 14^{\prime} \mathrm{N}$ ); $79 \%$ occurred more than $200 \mathrm{~km}$ offshore.

\section{Assemblages}

Assemblage analysis using the 2-way indicator species analysis program defined 2 distributional assemblages: 'island associated' and 'oceanic' (Table 2). The 15 'island-associated' species include 6 species with benthic adults (Octopus spp.), 6 species with mesopelagic adults (Abralia trigonura, Liocranchia reinhardti, L. valdiviae, Histioteuthis hoylei, Chiroteuthis picteti, and Enoploteuthis jonesi) and 3 species with epipelagic adults (Onychoteuthis $\mathrm{sp}$. C, Sthenoteuthis oualaniensis and Nototodarus hawailensis). Analysis of onshore-offshore abundance patterns showed that all 'island-associated' species decreased significantly in abundance with increasing distance offshore and had more than $50 \%$ of their paralarvae collected within $50 \mathrm{~km}$ of shore.

\section{DISCUSSION}

Paralarval distribution patterns provide further evidence that Abralia trigonura, Liocranchia reinhardti and Chiroteuthis pictetiare members of the Hawaiian Mesopelagic Boundary Community (MBC). However, the present data lend no support to Young's (1995) suggestion that Pterygioteuthis giardi is a probable facultative boundary species.

Paralarval catch data indicate that Liocranchia valdiviae, Histioteuthis hoylei, and Enoploteuthis jonesi are also probable $\mathrm{MBC}$ members. L. valdiviae adults were rarely caught near the Hawaiian Islands during $\mathrm{MBC}$ studies; however, the subadults of this deep-living species, unlike those of their congener, do not vertically migrate (Young 1978) and were thus mostly out of the sampling range of those studies. H. hoylei is widely distributed in 
the Pacific (Voss et al. 1998), but appears to spawn preferentially near land masses. Mature $H$. hoylei females collected off Japan occur in aggregations near an isolated oceanic rise, suggesting this species might spawn near the seafloor, as reported for $H$. miranda and $H$. celetaria pacifica (Nesis 1993a). H. reversa paralarvae also commonly occur near land masses (Clarke 1966). E. jonesi occurs in Hawaiian and equatorial waters (Burgess 1982) and has not been considered a member of the Hawaiian MBC (Young 1995); however, the

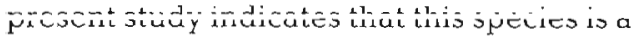
nearshore spawner. Some other Enoploteuthis species have been reported to spawn only over the slopes or in nearshore oceanic regions (Nesis 1993a, 1996).

The paralarvae of Sthenoteuthis oualaniensis, an abundant member of the oceanic nekton throughout the tropical and subtropical Indo-Pacific region (Roper et al. 1984, Young \& Hirota 1998), were also 'island associated'. Increased catches of S. oualaniensis paralarvae in nearshore waters also have been reported from the Saya de Malha Bank, Seychelles, and its slopes (Nesis 1993b) and the Arabian Sea (Piatkowski et al. 1993). Most mature S. oualaniensis females collected in the present study area occur along the windward slope of the islands (Suzuki et al. 1986, Young \& Hirota 1998), further suggesting that nearshore waters are favorable for spawning.

Some epipelagic squids near the Hawaiian Islands, including Sthenoteuthis oualaniensis and possibly Onychoteuthis sp. $C$, may migrate inshore before spawning. Nesis (1993a) has suggested that other oceanic squids (Todarodes sagittatus, Ornithoteuthis spp. and Pholidoteuthis spp.) similarly migrate to seamounts to spawn (Nesis 1993a). Inshore-offshore spawning migrations are common in the Loliginidae (Mangold 1987); these squid must find a solid substrate where they can attach their egg capsules.

Possibly good feeding conditions near the Hawaiian Islands could explain why some oceanic cephalopods appear to spawn preferentially nearshore. Young pelagic squids can consume 80 to $100 \%$ of their body weight per day (LaRoe 1971, Hurley 1976). Meeting such a high food requirement may be difficult, particularly in oligotrophic

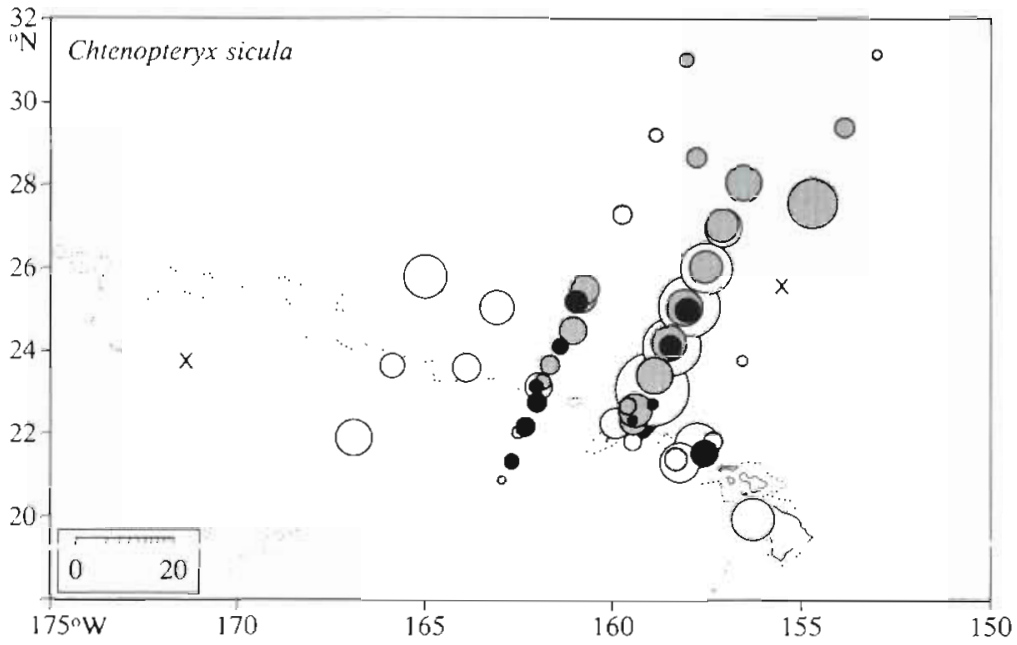

Fig. 8. Distribution and abundance of Chtenopteryx sicula. Symbols described in Fig. 1

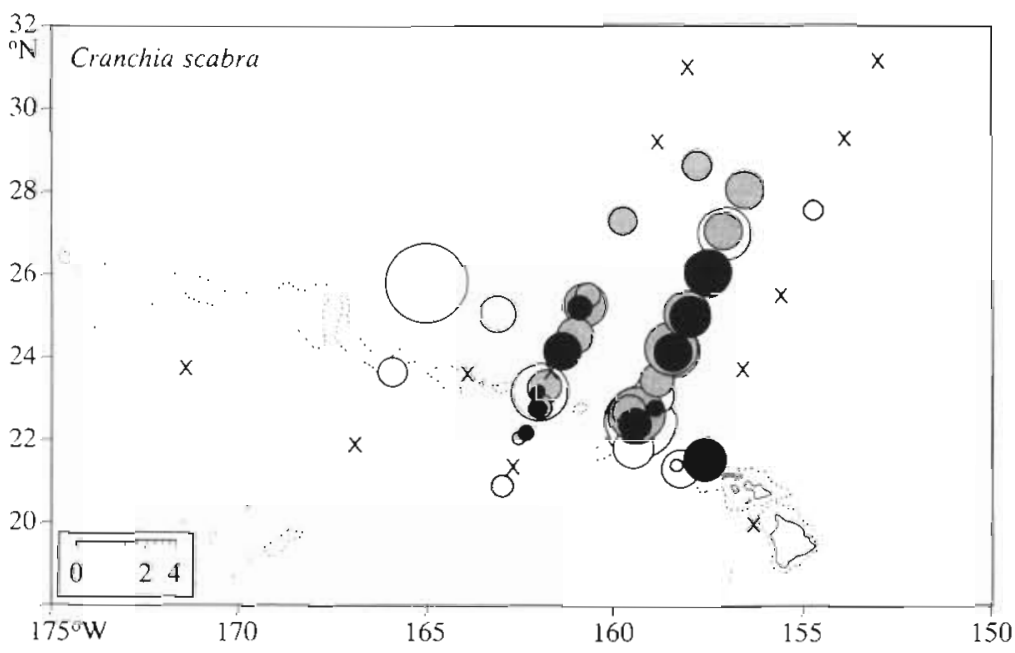

Fig. 9. Distribution and abundance of Cranchia scabra. Symbols described in Fig. 1

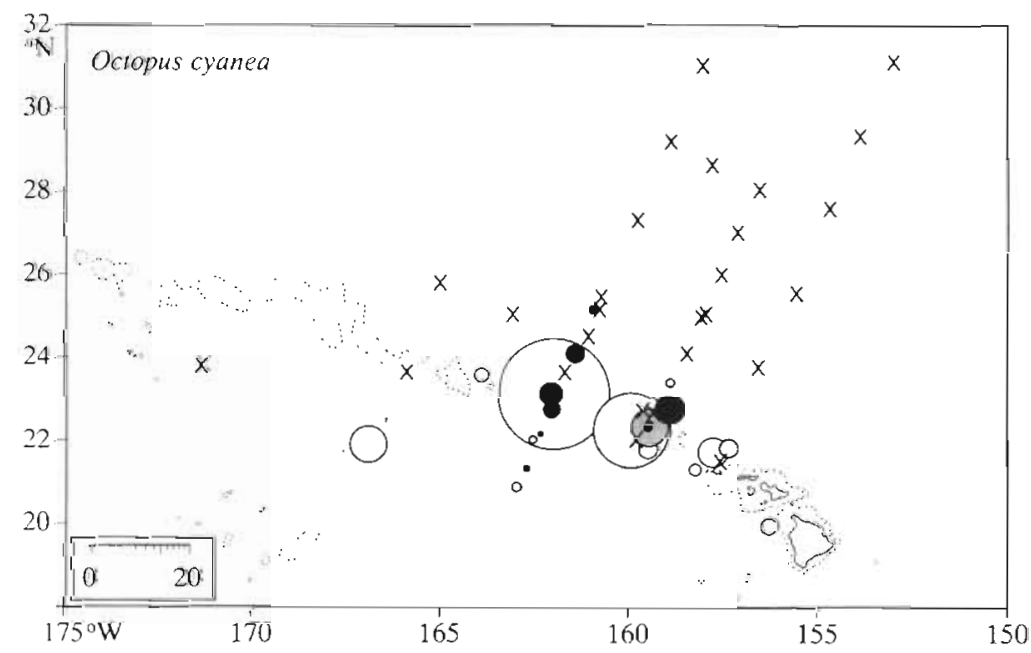

Fig. 10. Distribution and abundance of Octopus cyanea. Symbols described in Fig. 1 
Table 2. Paralarval assemblages identified from 5 plankton surveys near the Hawaiian Islands. Species groups within assemblages were determined with a 2 -way synthesis table technique (TWINSPAN). Only species with $\geq 15$ paralarvae collected were considered in the analyses. Literature references for species with letter designations are listed in Table 1

$\begin{array}{ll}\text { Island-associated assemblage } & \text { Oceanic assemblage } \\ \text { Abralia trigonura } & \text { Abraliopsis pacificus } \\ \text { Chiroteuthis picteti } & \text { Abraliopsis sp. A } \\ \text { Enoploteuthis jonesi } & \text { Ancistrocheirus lesueurii } \\ \text { Histioteuthis hoylei } & \text { Brachioteuthis sp. } \\ \text { Liocranchia reinhardti } & \text { Chiroteuthis sp. nov. } \\ \text { Liocranchia valdiviae } & \text { Cranchia scabra } \\ \text { Nototodarus hawailensis } & \text { Chtenopteryx sicula } \\ \text { Octopus cyanea } & \text { Eledonella pygmaea } \\ \text { Octopus Type B } & \text { Enoploteuthis higginsi } \\ \text { Octopus Type C } & \text { Enoploteuthis reticulata } \\ \text { Octopus Type E } & \text { Helicocranchia sp. B } \\ \text { Octopus Type H } & \text { Histioteuthis sp. C } \\ \text { Octopus Type I } & \text { Hyaloteuthis pelagica } \\ \text { Onychoteuthis sp. C } & \text { Leachia pacifica } \\ \text { Sthenoteuthis oualaniensis } & \text { Octopoteuthis nielseni } \\ & \text { Ommastrephes bartramii } \\ & \text { Onychoteuthis compacta } \\ & \text { Onykia sp. A } \\ & \text { Pholidoteuthis sp. } \\ & \text { Pterygioteuthis giardi } \\ & \text { Pterygioteuthis microlampas } \\ & \text { Pyroteuthis addolux } \\ & \text { Thysanoteuthis rhombus }\end{array}$

oceanic environments. The islands and seamounts that form the crest of the Hawaiian Ridge result in a number of mesoscale features, including seamount-induced biological and nutrient concentration disturbances (Boehlert \& Genin 1987), which could result in advantageous feeding conditions near the islands. Higher productivity and plankton biomass levels very near the Hawaiian Islands (Gilmartin \& Revelante 1974) may enhance paralarval survival for some species (see Dandonneau \& Charpy 1985). Unfortunately specific conditions that would provide a superior paralarval nursery ground are not known for these waters.

Waters near the Hawaiian Islands also may serve as favorable feeding grounds for spawning adults. Boehlert \& Mundy (1994) have suggested that this may be the case for spawning tuna. Pelagic species such as tuna and squid typically aggregate in waters near islands and banks (Uda \& Ishino 1958) and above seamounts (Inoue 1983, Yasui 1986) to feed. Increasing evidence indicates that some cephalopods, including Sthenoteuthis oualaniensis (Harman et al. 1989, Nigmatullin \& Laptikhovsky 1994), are multiple (i.e. iteroparous sensu lato) rather than single/terminal (i.e. semelparous sensu lato) spawners. Mature females spawning multiple batches of eggs on spawning grounds presumably must feed to survive and reproduce again.
In conclusion, the paralarval fauna near the Hawaiian Islands is the most diverse yet reported (Table 3 ). Species that showed evidence of increased spawning near the islands include not only those with bottom or nearshore spawning, such as Abralia trigonura, Nototodarus hawaiiensis, and Octopus spp., but also oceanic species not connected with the bottom of nearshore areas, such as Sthenoteuthis oualaniensis. Improved feeding conditions in waters near the archipelago might be one reason for the apparent increase in nearshore spawning by some oceanic species.

Acknowledgements. This study was supported by grants from the East-West Center (Honolulu) and the Japan Society for the Promotion of Science (Tokyo) awarded to J.R.B., from Hawaii Sea Grant (grant \# NA89AA-D-SG063) awarded to R.E.Y., and from the Joint Institute for Marine and Atmospheric Research (JIMAR) awarded to P.F. We appreciate reviews of the early manuscript by George Boehlert, ChungCheng Lu, Kir Nesis, Michael Vecchione and 2 anonymous reviewers. We also acknowledge the help of June Firing, Ilgiz Irnazarow, Takashi Okutani, Uwe Piatkowski, Stewart B. Reid and the officers and crews of the 'Hokusei Maru' and 'Townsend Cromwell' We dedicate this paper to the memory of KiYohiko Ishii.

\section{LITERATURE CITED}

Arkhipkin AI, Zheronkin YN, Loktionov YA, Shchetinnikov AS (1988) Fauna and distribution of pelagic cephalopods larvae in the Gulf of Guinea. Zool Zh 67(10):1459-1467 (in Russian with English abstract)

Berry SS (1914) The Cephalopoda of the Hawaiian Islands. Bull Bureau Fish 32(789):255-361

Boehlert GW, Genin A (1987) A review of the effects of seamounts on biological processes. In: Keating BH, Fryer P, Batiza R, Boehlert GW (eds) Seamounts, islands and atolls. Geophys Monogr Am Geophys Union 43:319-334

Boehlert GW. Mundy BC (1994) Vertical and onshore-offshore distributional patterns of tuna larvae in relation to physical habitat features. Mar Ecol Prog Ser 107:1-13

Bower JR (1994) Distribution of paralarvae of the squid Ommastrephes bartramii near the Hawaiian Archipelago. MSc thesis, Dept of Oceanography, University of Hawaii, Honolulu

Bower JR (1996) Estimated paralarval drift and inferred hatching sites for Ommastrephes bartramii (Cephalopoda Ommastrephidae) near the Hawaiian Archipelago. Fish Bull 94(3):398-411.

Boyle PR (1990) Cephalopod biology in the fisheries context Fish Res 8:303-321

Burgess LA (1982) Four new species of squid (Oegopsida: Enoploteuthis) from the Central Pacific and a description of adult Enoploteuthis reticulata. Fish Bull 80(4):703-734

Clarke MR (1966) A review of the systematics and ecology of oceanic squids. Adv Mar Biol 4:91-300

Dandonneau Y, Charpy L (1985) An empirical approach to the island mass effect in the south tropical Pacific based on sea surface chlorophyll concentrations. Deep-Sea Res 32: $707-721$

Dawe EG, Stephen SJ (1988) The cephalopod assemblage of the Gulf Stream System east of $60^{\circ} \mathrm{W}$. Malacologia 29(1): 235-245 
Table 3. Numbers of families and species of cephalopod paralarvae collected from different regions. "Octopods not reported

\begin{tabular}{|c|c|c|c|c|c|c|c|}
\hline & $\begin{array}{l}\text { Hawaiian } \\
\text { Islands }^{\mathrm{d}}\end{array}$ & $\begin{array}{l}\text { Pacific } \\
\text { Coast of } \\
\text { Japan }^{\text {b.c.d,e.t }}\end{array}$ & $\begin{array}{l}\text { Gulf Stream } \\
\text { east of } 60^{\circ} \mathrm{W}^{\mathrm{g}}\end{array}$ & $\begin{array}{l}\text { Gulf of } \\
\text { Guinea }^{h}\end{array}$ & $\begin{array}{l}\text { Northwest } \\
\text { Pacific }, i, j, l, m\end{array}$ & $\begin{array}{l}\text { Arabian } \\
\text { Sea }^{\mathrm{n}, 0 . p}\end{array}$ & $\begin{array}{l}\text { California } \\
\text { Current }\end{array}$ \\
\hline \multicolumn{8}{|l|}{ Order Octopoda } \\
\hline Alloposidae & 0 & & 1 & 0 & 0 & 0 & \\
\hline Argonautidae & 1 & & 1 & 1 & 0 & 0 & \\
\hline Bolitaenidae & 1 & & 0 & 0 & 1 & 1 & \\
\hline Octopodidae & 9 & & 2 & 3 & 1 & 1 & \\
\hline Ocythoidae & 0 & & 0 & 1 & 0 & 0 & \\
\hline Tremoctopodidae & 1 & & 0 & 0 & 0 & 0 & \\
\hline \multicolumn{8}{|l|}{ Order Sepioidea } \\
\hline Idiosepiidae & 0 & 1 & 0 & 0 & 0 & 0 & 0 \\
\hline Seninlidas & 1 & $\hat{3}$ & $\bar{u}$ & $u$ & 0 & 0 & 0 \\
\hline \multicolumn{8}{|l|}{ Order Teuthida } \\
\hline Ancistrocheiridae & 1 & 1 & 0 & 1 & 0 & 0 & 0 \\
\hline Bathyteuthididae & 0 & 0 & 0 & 0 & 0 & 1 & 0 \\
\hline Brachioteuthididae & 1 & 1 & 1 & 1 & 1 & 0 & 0 \\
\hline Chiroteuthididae & 4 & 1 & 1 & 0 & 0 & 1 & 1 \\
\hline Chtenopterygidae & 1 & 1 & 1 & 1 & 0 & 1 & 1 \\
\hline Cranchidae & 7 & 7 & 3 & 3 & 1 & 2 & 4 \\
\hline Cycloteuthididae & 1 & 0 & 0 & 1 & 0 & 0 & 0 \\
\hline Enoploteuthididae & 7 & 4 & 3 & 2 & 2 & 3 & 1 \\
\hline Gonatidae & 0 & 2 & 2 & 0 & 9 & 0 & 1 \\
\hline Histioteuthididae & 4 & 0 & 1 & 1 & 1 & 0 & 1 \\
\hline Joubiniteuthididae & 0 & 0 & 1 & 0 & 0 & 1 & 0 \\
\hline Lepidoteuthididae & 1 & 0 & 0 & 0 & 0 & 0 & 0 \\
\hline Loliginidae & 0 & 2 & 0 & 0 & 0 & 0 & 1 \\
\hline Lycoteuthididae & 0 & 0 & 1 & 0 & 0 & 0 & 0 \\
\hline Mastigoteuthididae & 1 & 0 & 1 & 1 & 0 & 0 & 0 \\
\hline Neoteuthididae & 0 & 0 & 0 & 0 & 0 & 0 & 0 \\
\hline Octopoteuthididae & 2 & 1 & 1 & 0 & 1 & 1 & 1 \\
\hline Ommastrephidae & 4 & 6 & 1 & 2 & 1 & 2 & 1 \\
\hline Onychoteuthididae & 5 & 3 & 2 & 3 & 1 & 1 & 1 \\
\hline Pholidoteuthididae & 1 & 0 & 0 & 0 & 0 & 0 & 0 \\
\hline Psychroteuthididae & 0 & 0 & 0 & 0 & 0 & 0 & 0 \\
\hline Pyroteuthididae & 3 & 1 & 4 & 2 & 0 & 0 & 1 \\
\hline Thysanoteuthididae & 1 & 1 & 0 & 1 & 1 & 0 & 0 \\
\hline No. of families & 21 & $15^{\circ}$ & 17 & 15 & 11 & 11 & $11^{\circ}$ \\
\hline No. of species & 57 & $35^{\circ}$ & 27 & 24 & 20 & 15 & $14^{\circ}$ \\
\hline \multicolumn{8}{|c|}{ 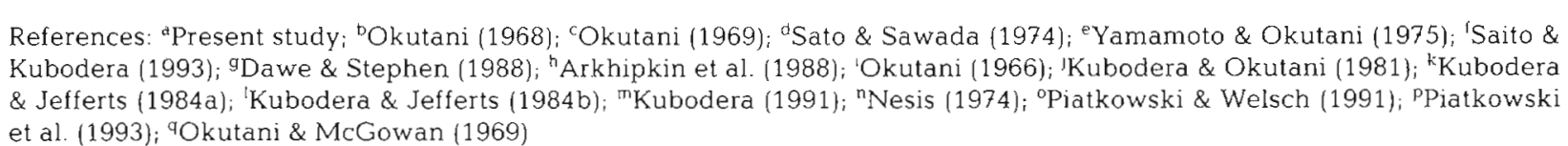 } \\
\hline
\end{tabular}

Gauch HG Jr, Whittaker RH (1981) Hierarchial classification of community data. J Ecol 69(2):537-557

Gilmartin M, Revelante N (1974) The 'island mass effect' on the phytoplankton and primary production of the Hawaiian Islands. J Exp Mar Biol Ecol 16:181-204

Harman RF, Young RE (1985) The larvae of ommastrephid squids (Cephalopoda, Teuthoidea) from Hawailan waters. Vie Milieu 35(3/4):211-222

Harman RF, Young RE, Reid SB, Mangold KM, Suzuki T, Hixon RF (1989) Evidence for multiple spawning in the tropical oceanic squid Stenoteuthis oualaniensis (Teuthoidea: Ommastrephidae). Mar Biol 101:513-519

Hill MO (1979) TWINSPAN - a FORTRAN program for arranging multivariate data in an ordered two-way table by classification of the individuals and attributes. Ecology and Systematics, Cornell University, Ithaca, NY

Hurley AC (1976) Feeding behavior, food consumption, growth, and respiration of the squid Loligo opalescens raised in the laboratory. Fish Bull 74(1):176-182

Inoue M (1983) Exploitation of fishing grounds of southward migrants of fall skipjacks in the northwestern Pacific Ocean. J Fac Mar Sci Technol Tokai Univ 17:121-130 (in Japanese with English abstract)

Kubodera T (1991) Distribution and abundance of the early life stages of octopus, Octopus dofleini Wulker 1910 in the North Pacific. Bull Mar Sci 49(1-2):235-243

Kubodera T, Jefferts K (1984a) Distribution and abundance of the early life stages of squid, primarily Gonatidae (Ceph- 
alopoda, Oegopsida), in the northern North Pacific (Part 1). Bull Natl Sci Mus Ser A (Zool) 10(3):91-106

Kubodera T, Jefferts K (1984b) Distribution and abundance of the early life stages of squid, primarily Gonatidae (Cephalopoda, Oegopsida), in the northern North Pacific (Part 2). Bull Natl Sci Mus Ser A (Zool) 10(4):165-193

Kubodera T, Okutani T (1981) The systematics and identification of larval cephalopods from the northern North Pacific. Res Inst N Pac Fish Hokkaido Univ Spec Vol, p 131-159

LaRoe ET (1971) The culture and maintenance of the loliginid squids Sepioteuthis sepioidea and Doryteuthis plei. Mar Biol 9(1):9-25

Mangold K (1987) Reproduction. In: Boyle PR (ed) Cephalopod life cycles, Vol 2. Academic Press Inc, London, p $157-200$

McCune B, Mefford MJ (1997) PC-ORD. Multivariate analysis of ecological data, Version 3.0. MjM Software Design, Gleneden Beach, OR

Nesis KN (1974) Cephalopod larvae in the western Arabian Sea. Oceanology 14(3):441-445

Nesis K (1993a) Cephalopods of seamounts and submarine ridges. In: Okutani T, O'Dor RK, Kubodera T (eds) Recent advances in cephalopod fisheries biology. Tokai University Press, Tokyo, p 365-373

Nesis K (1993b) Population structure of oceanic ommastrephids, with particular reference to Sthenoteuthis oualaniensis: a review. In: Okutani T, O'Dor RK, Kubodera T (eds) Recent advances in cephalopod fisheries biology. Tokai University Press, Tokyo, p 375-383

Nesis KN (1996) Mating, spawning, and death in oceanic cephalopods: a review. Ruthenica 6(1):23-64

Nigmatullin CM, Laptikhovsky VV (1994) Reproductive strategies in the squids of the family Ommastrephidae (preliminary report). Ruthenica 4(1):79-82

Okutani T (1966) Studies on early life history of decapodan Mollusca-II. Planktonic larvae of decapodan cephalopods from the northern North Pacific in summer seasons during 1952-1959. Bull Tokai Reg Fish Res Lab 45:61-79

Okutani T (1968) Studies on early life history of decapodan Mollusca-III. Systematics and distribution of larvae of decapod cephalopods collected from the sea surface on the Pacific coast of Japan 1960-1965. Bull Tokai Reg Fish Res Lab 55:9-57

Okutani T (1969) Studies on early life history of decapodan Mollusca - IV. Bull Tokai Reg Fish Res Lab 58:83-96

Okutani T, McGowan JA (1969) Systematics, distribution, and abundance of the epiplanktonic squid (Cephalopoda, Decapoda) larvae of the California Current April 1.954 - March 1957. Bull Scripps Inst Oceanogr Univ Calif 14:1-90

Piatkowski U, Welsch W (1991) On the distribution of pelagic cephalopods in the Arabian Sea. Bull Mar Sci 49(1-2): $186-198$

Piatkowski U, Welsch W, Ropke A (1993) Distribution patterns of the early life stages of pelagic cephalopods in three geographically different regions of the Arabian Sea. In: Okutani T, O'Dor RK, Kubodera T (eds) Recent advances in cephalopod fisheries biology. Tokai University Press, Tokyo, p 417-431

Reid SB, Hirota J, Young RE, Hallacher LE (1991) Mesopelagic-boundary community in Hawaii: micronekton at the interface between neritic and oceanic systems. Mar Biol 109:427-440

Roper CFE, Sweeney MJ, Nauen CE (1984) FAO species catalogue. Vol 3. An annotated and illustrated catalogue of

Editorial responsibility: Kenneth Sherman (Contributing Editor), Narragansett, Rhode Island, USA. species of interest to fisheries. Cephalopods of the world FAO Fish Synop 125, FAO, Rome

Saito H, Kubodera T (1993) Distribution of ommastrephid rhynchoteuthion paralarvae (Mollusca, Cephalopoda) in the Kuroshio region. In: Okutani T, O'Dor RK, Kubodera $T$ (eds) Recent advances in cephalopod fisheries biology. Tokai University Press, Tokyo, p 457-466

Sato $K$. Sawada $T$ (1974) Studies on larvae of decapod cephalopods in the waters of the Izu Peninsula - I. Identification of larvae. Bull Shizuoka Prefect Fish Exp Stn 8: 11-21 (in Japanese)

Smith PE, Richardson SL (1977) Standard techniques for pelagic fish egg and larva surveys. FAO Fish Tech Pap 175, FAO, Rome

Suzuki T, Yamamoto S, Ishii K, Matsumoto WM (1986) On the flying squid Stenotuthis [sic] oualaniensis (Lesson) in Hawaiian waters. Bull Fac Fish Hokkaido Univ 37(2): 111-123

Uda M. Ishino M (1958) Enrichment pattern resulting from eddy systems in relation to fishing grounds. J Tokyo Univ Fish 44:105-129

Vecchione M (1987) Juvenile ecology. In: Boyle PR (ed) Cephalopod life cycles, Vol 2. Academic Press Inc, London, p $61-84$

Voss NA, Nesis KN, Rodhouse PG (1998) The cephalopod family Histioteuthidae (Oegopsida): systematics, biology, and biogeography. Smithson Contrib Zool 586:293-372

Yamamoto K, Okutani T (1975) Studies on early life history of decapodan Mollusca-V. Systematics and distribution of epipelagic larvae of decapod cephalopods in the southwestern waters of Japan during the summer in 1970. Bull Tokai Reg Fish Res Lab 83:45-96

Yasui M (1986) Albacore. Thunnus alalunga, pole-and-line fishery around the Emperior [sic] Seamounts. In: Uchida RN, Hayasi S, Boehlert GW (eds) Environment and resources of seamounts in the North Pacific. NOAA Tech Rep NMFS 43:37-40

Young RE (1978) Vertical distribution and photosensitive vesicles of pelagic cephalopods from Hawaian waters. Fish Bull 76(3):583-615

Young RE (1991) Chiroteuthid and related paralarvae from Hawaiian waters. Bull Mar Sci 49(1-2):162-185

Young RE (1.995) Aspects of the natural history of pelagic cephalopods of the Hawaiian mesopelagic-boundary region. Pac Sci 49(2):143-155

Young RE. Harman RF (1985) Early life history stages of enoploteuthin squids (Cephalopoda: Teuthoidea: Enoploteuthidae) from Hawaiian waters. Vie Milieu 35(3/4):181-201

Young RE, Harman RF (1987) Descriptions of the larvae of three species of the Onychoteuthis banksii complex from Hawaiian waters. Veliger 29(3):313-321.

Young RE, Harman. RF (1989) Octopodid paralarvae from Hawaiian waters. Veliger 32(2):152-165

Young RE. Hirota J (1990) Description of Ommastrephes bartramii (Cephalopoda: Ommastrephidae) paralarvae with evidence of spawning in Hawaiian waters. Pac Sci 44(1): $71-80$

Young RE, Hirota J (1998) Review of the ecology of Sthenoteuthis oualaniensis near the Hawaitan Archipelago. In Okutani $T$ (ed) Contributed papers to international symposium on large pelagic squids. Japan Marine Fishery Resources Research Center, Tokyo, p 131-143

Young RE, Harman RF, Mangold KM (1985) The eggs and larvae of Brachioteuthis sp. (Cephalopoda: Teuthoidea) from Hawailan waters. Vie Milieu 35(3/4):203-209

Submitted: September 16, 1998; Accepted: January 7, 1999

Proofs received from author(s): July 29, 1999 